\title{
Conocimientos matemáticos del profesorado de la Escuela Infantil (0-3 años): efecto en el diseño de espacios para desarrollar las matemáticas informales
}

\author{
Gloria Olmos Martínez ${ }^{1}$ y Ángel Alsina ${ }^{2 *}$ \\ ${ }^{1}$ Coordinadora Pedagógica de las Escuelas Infantiles Municipales de Vic, ${ }^{2}$ Universidad de Girona
}

\author{
PALABRAS CLAVE \\ Conocimiento matemático \\ del profesorado \\ Matemáticas informales \\ Enseñanza de las matemáticas \\ Espacios y materiales \\ Escuela infantil
}

\begin{abstract}
RESUMEN
En este estudio se analizan los conocimientos matemáticos del profesorado de la Escuela Infantil para favorecer el desarrollo de los primeros conocimientos matemáticos intuitivos e informales de los niños y niñas de 0 a 3 años, a través del diseño de los espacios y materiales. Se ha diseñado un estudio cuasi-experimental (Pre-Post) en el que, antes y después de una actividad de formación, se ha administrado el Cuestionario "Conocimientos Didáctico-Matemáticos en la Escuela Infantil" (CDM 0-3) a 28 profesionales. Los resultados obtenidos muestran cambios significativos en los conocimientos matemáticos $y$, por consiguiente, en el diseño de los espacios y materiales que se ofrecen a los niños y las niñas de 0 a 3 años, incrementando el contenido y sentido matemático. Se concluye que es imprescindible una formación inicial y continua de calidad que garantice que los profesionales de la Escuela Infantil tengan los conocimientos matemáticos necesarios para diseñar espacios ricos desde un punto de vista matemático.
\end{abstract}

\section{Mathematical knowledge of Nursery School teachers (0-3 years): effect on the design of spaces to develop intuitive mathematics}

Teachers' mathematical

knowledge

Informal mathematics

Mathematics teaching

Spaces and materials

Infant school

\begin{abstract}
This study analyses the mathematical knowledge of nursery school teachers in order to promote the development of the first intuitive and informal mathematical knowledge of children under 3 years of age, through the design of spaces and materials. A quasi-experimental study (Pre-Post) was designed, where before and after a training activity, the questionnaire "Didactic-Mathematical Knowledge in Nursery School" (CDM 0-3) was administered to 28 professionals. The results obtained show significant changes in mathematical knowledge and, consequently, in the design of spaces and materials offered to children under 3 years of age, increasing mathematical content and meaning. It is concluded that it is essential to provide initial and continuous quality training to ensure that nursery school professionals have the necessary mathematical knowledge to design spaces that are rich from a mathematical point of view.
\end{abstract}

\section{Universidad de Girona}

Autor de correspondencia: * Ángel Alsina; angel.alsina@udg.edu

Recibido: 13/02/2021 - Aceptado: 07/05/2021

Revista de Formación del Profesorado e Investigación Educativa

Facultad de Formación del Profesorado y Educación

Universidad de Oviedo

Enero - Diciembre 2021

ISSN: $2340-4728$

(c) (1) () $\odot$ Esta obra está bajo una licencia internacional Creative Commons

Atribución-NoComercial-SinDerivadas 4.0. 


\section{Introducción}

La Escuela Infantil (0-3 años) es la gran olvidada tanto de la investigación como de la Administración educativa. A pesar de que la mayoría de autores y organismos coinciden en que este periodo es de gran importancia (Bueno, 2019; Hoyuelos, 2010; UNICEF, 2017;), sigue vigente la paradoja entre el valor y la importancia de los tres primeros años de vida para el desarrollo humano y las políticas educativas, que continúan sin favorecer el primer ciclo educativo, perpetuando diferencias muy significativas con los otros niveles educativos. Hoyuelos (2010) y Vila (2018) señalan que una de estas diferencias es, por ejemplo, la retribución que reciben los profesionales de la Escuela Infantil, con salarios más bajos que el resto de profesionales de la educación, infravalorando así la función que realizan.

Esta dualidad entre el papel de la educación en la primera infancia y el escaso reconocimiento de las políticas educativas escenifica la necesidad de la investigación educativa en la Escuela Infantil, como la oportunidad de seguir validando y reconociendo la calidad de la educación en la primera infancia. Desde este marco, este artículo se focaliza en la investigación en educación matemática infantil y, más concretamente, en el análisis de los conocimientos matemáticos de los profesionales de la Escuela Infantil para promover el desarrollo del pensamiento matemático de los niños y las niñas de 0 a 3 años, como una de las habilidades que contribuyen a su desarrollo integral (Alsina, 2015). En este sentido, se asume que la educación matemática en la Escuela Infantil requiere contar con profesionales formados y conscientes de la importancia de ofrecer contextos educativos de calidad que promuevan el desarrollo de acciones asociadas a las primeras matemáticas (Alsina, 2006, 2015; Alsina y Berciano, 2018; Alsina y León, 2016; Alsina y Martínez, 2016; Björklund y Barendregt, 2016; de Castro, 2011; de Castro et al., 2015; Clements y Sarama, 2015; Edo, 2012; Varol et al., 2012;). En la Figura 1 se muestran tres finalidades de esta línea de investigación en torno a los conocimientos de los profesionales de la Escuela Infantil para promover el desarrollo del pensamiento matemático:

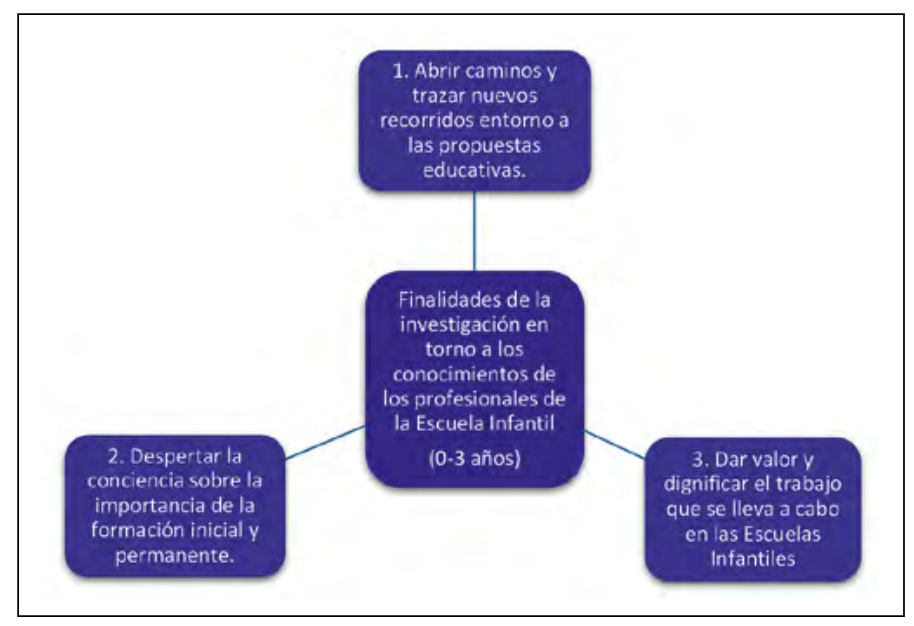

Fuente: Elaboración propia

Figura 1. Finalidades de la investigación en torno a los conocimientos de los profesionales de la Escuela Infantil para promover el desarrollo del pensamiento matemático

A continuación, se justifican estas tres finalidades:

1) Abrir caminos y trazar nuevos recorridos entorno a las propuestas educativas: de este modo, la investigación y la innovación van de la mano, contribuyendo al desarrollo de prácticas mejoradas mediante recursos, ideas y propuestas para implementar en las aulas; y, a la vez, generando movimientos, reflexiones, cambios e innovaciones en los diseños de los espacios y materiales de las diferentes propuestas de experimentación y juego.
2) Despertar la conciencia sobre la importancia de la formación inicial y permanente: la investigación y la formación como puentes para romper las barreras que existen en torno a las creencias de que las matemáticas son conocimientos propios de otros niveles superiores, junto con la idea de que las matemáticas que se abordan en la Escuela Infantil carecen de importancia, son sencillas y, por tanto, cualquiera las puede enseñar (Castro y Castro, 2016). Asumimos que los profesionales que trabajan en la Escuela Infantil deben dominar los contenidos y procesos propios de esta área, de manera que puedan ofrecer una respuesta educativa de calidad. Por esta razón, defendemos una formación inicial y, en su defecto, permanente, que ofrezca los conocimientos disciplinares y didácticos necesarios.

3) Dar valor y dignificar el trabajo que se lleva a cabo en las Escuelas Infantiles: este período se interpreta a menudo como el último eslabón (en lugar del primero) y se infravalora su función educativa $\mathrm{y}$, aún más, si se trata del conocimiento matemático. Existe una creencia, todavía muy arraigada, de considerar que las matemáticas son propias de edades más avanzadas, a pesar de que diversos autores vienen señalando que las "primeras matemáticas" son la base necesaria para los aprendizajes posteriores (Alsina, 2006, 2015; Baroody, 1987; de Castro, 2011; Clements y Sarama, 2015; Edo, 2012; Geist, 2014).

Con base en estos antecedentes, en este estudio -que forma parte de una investigación de mayor envergadura- se presenta un primer análisis de los conocimientos matemáticos de los profesionales de la Escuela Infantil (0-3 años) para favorecer el desarrollo de las matemáticas intuitivas, a través del diseño de los espacios y materiales.

\section{Marco teórico}

Considerando las finalidades de este estudio, en primer lugar, se presenta una breve síntesis de los modelos de conocimiento del profesorado para enseñar matemáticas (Ball et al., 2008; Carrillo et al., 2018; Godino et al., 2017;), haciendo especial hincapié en el Modelo "Conocimientos para Enseñar Matemáticas en Educación Infantil" (CEM-EI), que es el que se asume para analizar los conocimientos matemáticos de los profesionales de la Escuela Infantil (Alsina y Delgado, 2021); y, en segundo lugar, se realiza una revisión de estudios acerca del diseño de espacios y materiales en la Escuela Infantil (Abad, 2006; Alsina, 2015; Azkona y Hoyuelos, 2011; Bassedas et al., 2006; de Castro y Quiles, 2014; de Castro et al., 2009; Edo, 2012; Galardini, 2010; Goldschmied y Jackson, 2007; Jubete, 2004; Malaguzzi, 2001; Rinaldi, 2009).

Conocimientos de los profesionales de la Escuela Infantil para enseñar matemáticas intuitivas

Alsina $(2019,2020 a, 2020 b)$ subraya que, en el marco de la investigación en educación matemática infantil, una de las agendas de investigación que necesita más atención está relacionada con el análisis de los conocimientos que un profesional debería tener para que su práctica de enseñanza de las matemáticas sea lo más idónea posible.

En este sentido, han surgido distintos modelos de conocimiento para enseñar matemáticas entre los cuales destacan el Mathematical Knowledge for Teaching (MKT), que considera un conjunto de conocimientos y habilidades que requiere el profesorado para gestionar las tareas y los problemas recurrentes en la enseñanza de las matemáticas (Ball et al., 2008); el Modelo de Conocimientos y Competencias Didáctico-Matemáticas (CCDM) de Godino et al. (2017), para analizar, interpretar, caracterizar y categorizar los conocimientos que pone en juego el profesorado al enseñar un determinado contenido matemático; o el Modelo del Conocimiento Especializado del Profesor de Matemáticas (MTSK), que asume que todo el conocimiento que es útil para el profesorado en el contexto de enseñanza y aprendizaje de las matemáticas es especializado (Carrillo et al., 2018).

La mayoría de estudios desarrollados desde estos distintos modelos se han focalizado en profesorado de matemáticas de pri- 
maria y secundaria, mientras que las investigaciones sobre el conocimiento del profesorado de infantil son escasas (Charalambous y Pitta-Pantazi, 2016). Para subsanar este déficit, Alsina y Delgado (2021) han desarrollado el Modelo "Conocimientos para Enseñar Matemáticas en Educación Infantil" (CEM-EI), que está constituido por dos dominios: Conocimiento Matemático en Educación Infantil (CM-EI), que se refiere al conocimiento matemático específico y estructurado que permita promover el desarrollo de conocimientos y habilidades matemáticas de los niños y niñas, y el Conocimiento Didáctico de las Matemáticas en Educación Infantil (CDM-EI), que se refiere a los conocimientos psicopedagógicos sobre cómo aprenden matemáticas los niños y niñas y cómo se enseñan las matemáticas en Educación Infantil. Como se aprecia en la Figura 2, cada dominio incluye tres subdominios.

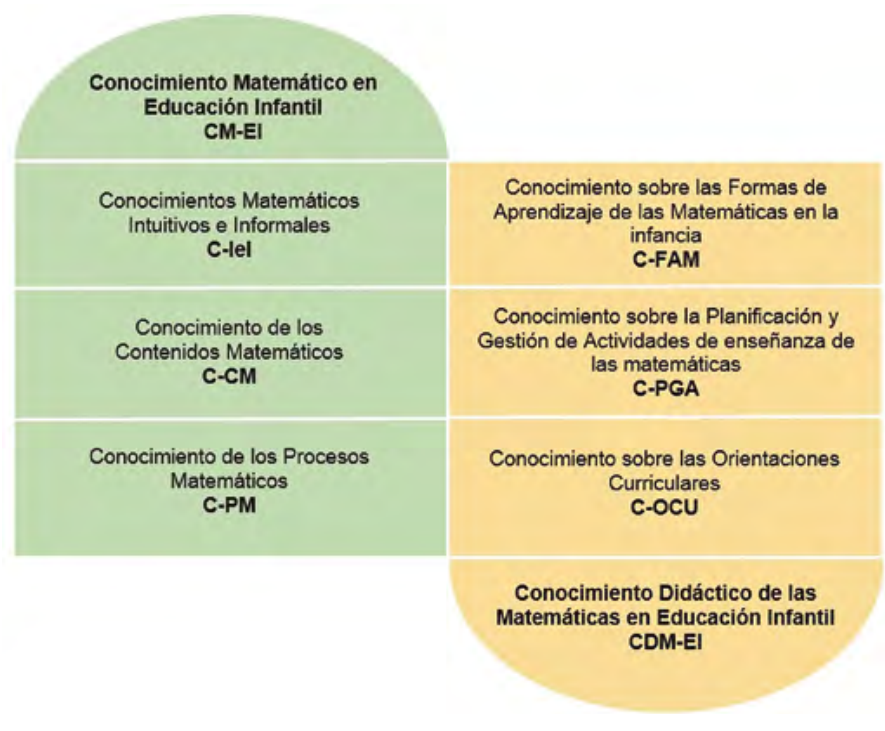

Fuente: Alsina y Delgado (2021, p. 6)

Figura 2. Modelo "Conocimientos para Enseñar Matemáticas en Educación Infantil"

El dominio CM-EI incluye tres subdominios: 1) Conocimientos Matemáticos Intuitivos e Informales (C-IeI), que se refiere al conocimiento acerca de las primeras matemáticas que los niños y niñas aprenden en situaciones informales de exploración del entorno, manipulación y juegos, que han sido ampliamente definidas por autores como Alsina (2015), Clements y Sarama (2015) y Geist (2014), entre otros, y que son un eslabón imprescindible para el acceso a las matemáticas más formales (NCTM, 2003); 2) el Conocimiento de los contenidos matemáticos (C-CM), que se refiere al conocimiento de los temas matemáticos incluidos en cada bloque de contenidos del currículo de Educación Infantil (álgebra temprana, números y operaciones, geometría, medida, y estadística y probabilidad), junto con el conocimiento de relaciones entre temas matemáticos ubicados en el mismo bloque de contenidos (conexiones intraconceptuales), así como relaciones entre temas pertenecientes a diferentes bloques de contenidos (conexiones interconceptuales); y, finalmente, 3) el Conocimiento de los Procesos Matemáticos (C-PM), que se refiere al conocimiento sobre qué es un problema; qué estrategias y heurísticas se pueden utilizar para resolver un problema; cómo, porqué y para qué se argumenta; cuáles son los diferentes tipos de razonamiento; o cómo se utilizan los símbolos y el lenguaje para comunicar ideas matemáticas (Alsina, 2012; NCTM, 2003).

El dominio Conocimiento Didáctico (CDM-EI) incluye también tres subdominios: 1) el Conocimiento sobre las Formas de Aprendizaje de las Matemáticas en la Infancia (C-FAM), que se refiere a los conocimientos sobre cómo aprenden matemáticas los niños y niñas de 0 a 6 años; 2) el Conocimiento sobre la Planifica- ción y Gestión de Actividades de enseñanza de las matemáticas (C-PGA), que se refiere a los conocimientos sobre el diseño y la implementación de actividades que permitan promover una educación matemática inclusiva en Educación Infantil; y, finalmente, 3) el Conocimiento sobre las Orientaciones Curriculares (C-OCU), que se refiere a los conocimientos acerca del currículo, tanto en lo que respecta a las bases psicopedagógicas sobre el proceso de enseñanza-aprendizaje, la organización de la Educación Infantil por áreas en lugar de asignaturas (conocimiento de uno mismo y autonomía personal, conocimiento del entorno y comunicación y lenguajes) y la evaluación (inicial y formativa, principalmente) como elemento indisociable del proceso de enseñanza-aprendizaje.

Los espacios de exploración y juego con sentido matemático en la Escuela Infantil

Los profesionales de la Escuela Infantil van creando entornos y seleccionando experiencias con orígenes e influencias didácticas diversas, con el fin de crear propuestas coherentes que reflejen una concepción profundamente educativa del ciclo de 0 a 3 años (de Castro et al., 2015). En este sentido, múltiples autores manifiestan la importancia de ofrecer espacios multisensoriales con elementos ricos, variados, bien presentados, que respondan a la necesidad innata de explorar, investigar, desarrollar, conocer, etc. y, a su vez, contribuyan al desarrollo global de sus capacidades y aptitudes a través del juego y la exploración (Abad, 2006; Alsina, 2015; Azkona y Hoyuelos, 2011; Bassedas et al., 2006; de Castro y Quiles, 2014; de Castro et al., 2009; Edo, 2012; Galardini, 2010; Goldschmied y Jackson, 2007; Jubete, 2004; Malaguzzi, 2001; Rinaldi, 2009). Estos espacios, como dice Rinaldi (2009), deben garantizar la seguridad y la personalización del niño, respetando su momento perceptivo, cognitivo y emocional, cuidando minuciosamente los diferentes ambientes que se le pueden ofrecer (refugios, espacios tranquilos, de exploración, de movimiento, de creación...), las superficies, los mobiliarios y los materiales, para que sepan salvaguardar al máximo las necesidades y deseos de autonomía y los tiempos, rutinas e identidades diversas. De forma sintética, estos autores hacen referencia a diferentes tipos de espacios, que se describen a continuación y se ilustran con imágenes tomadas en la red de Escuelas Infantiles Municipales de Vic (Catalunya, España):

- Los espacios con diferentes materiales de exploración (Figura 3): se trata de espacios con materiales de origen natural, normalmente no estructurados ni comercializados, ordenados y expuestos al alcance de los niños y las niñas de 0 a 3 años (objetos de madera, de metal, de ropa, etc.). Con ellos, pueden descubrir y explorar las cualidades sensoriales, las magnitudes, las cantidades discretas o las posiciones y las formas a través de acciones como identificar, agrupar, aparear, clasificar, observar los cambios, etc.

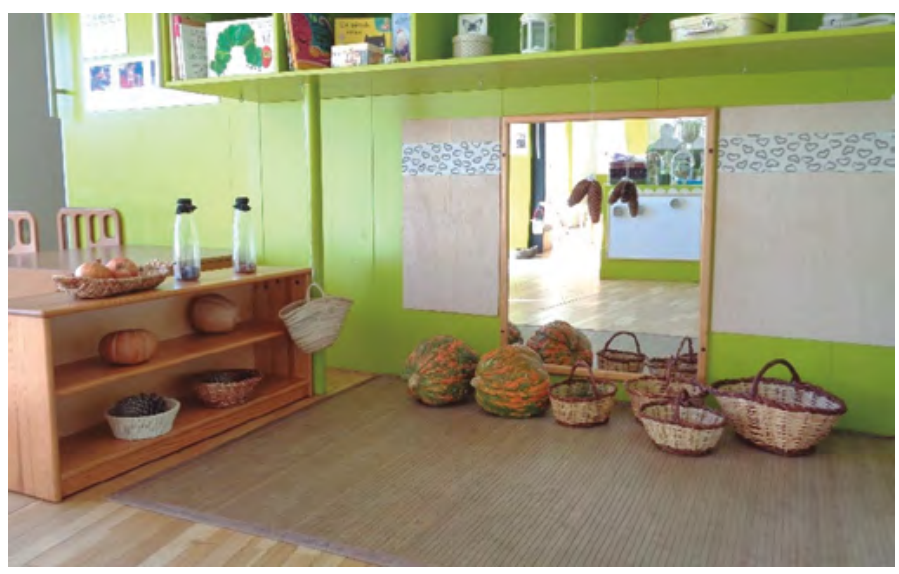

Fuente: Elaboración propia

Figura 3. Espacio con diferentes materiales de exploración 
- Los espacios de juego heurístico (Figura 4): parten de la exploración de los niños y las niñas, donde el reto es descubrir que pueden hacer con los diferentes elementos desarrollando acciones como poner, sacar, vaciar, llenar, tapar, destapar, encajar, alinear, estirar, agrupar, desagrupar, colgar, descolgar, etc.

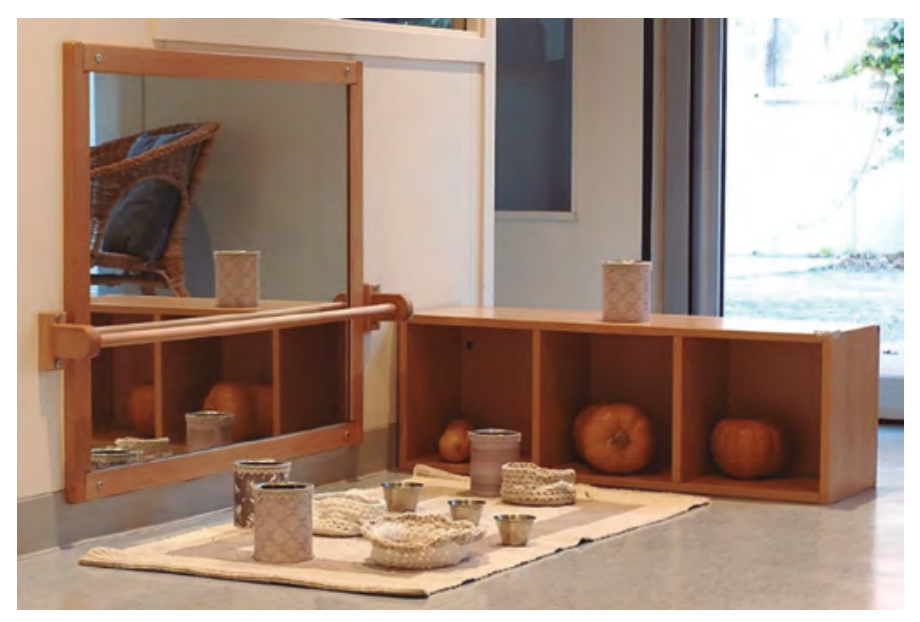

Fuente: Elaboración propia

Figura 4. Espacio de juego heurístico

- Los espacios con mesas de experimentación (Figura 5): la finalidad es hacer hipótesis y comprobar, a partir del material inespecífico, que se dispone dentro de una bandeja lo suficientemente amplia para que contenga además los diferentes contenedores y los diferentes utensilios para favorecer la experimentación.

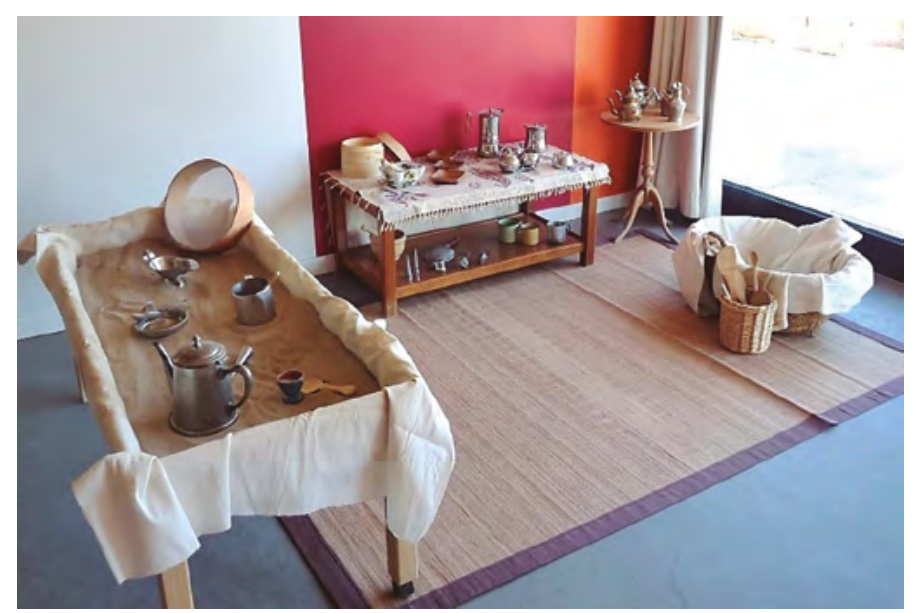

Fuente: Elaboración propia

Figura 5. Espacio con mesas de experimentación

- Los espacios de juego simbólico (Figura 6): diferentes ambientes que reproducen situaciones cotidianas del hogar o de la vida práctica (la cocinita, la zona de baño para las muñecas y cambiador de ropa, una tienda de alimentos o un taller mecánico, entre otros). Estos espacios contienen muchos complementos para favorecer una propuesta rica de juego como escenario para que, los niños y las niñas, a través del movimiento, el juego libre, la expresión y comunicación, reproduzcan simbólicamente e imiten situaciones cotidianas como cocinar, dar de comer, bañar, vestir los muñecos o a sí mismos y a sus compañeros de juego.

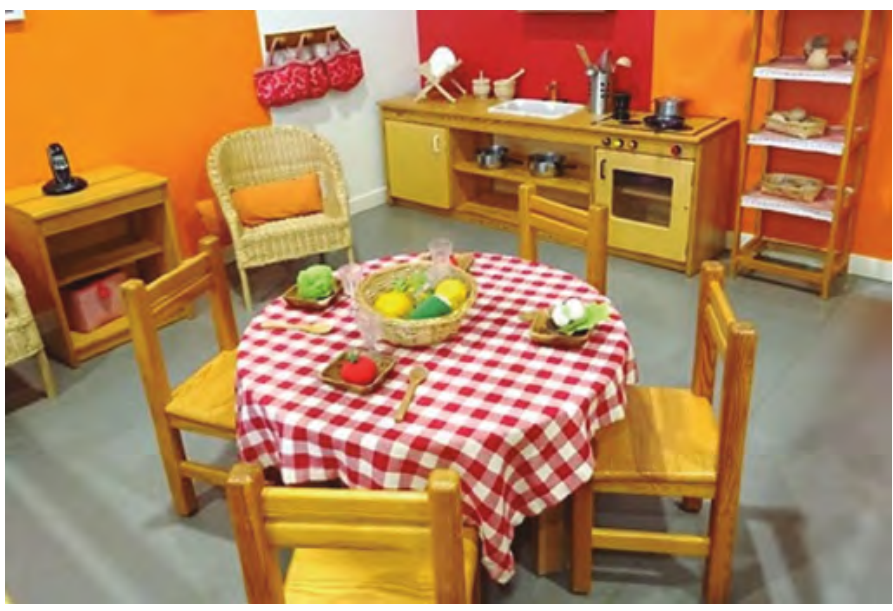

Fuente: Elaboración propia

Figura 6. Espacio de juego simbólico

- Los espacios de movimiento (Figura 7): diseñados específicamente como retos a conquistar con todo el cuerpo, como pueden ser rampas y toboganes, cajones de diferentes tamaños para subirse encima o ponerse dentro, túneles donde esconderse, bloques gigantes donde subirse o intentar arrastrar, hamacas o columpios para balancearse, colchonetas donde tumbarse y rodar, gatear y arrastrarse, pelotas gigantes y pelotas pequeñas, telas de diferentes tamaños y colores, puentes y cilindros, etc. Todos ellos, elementos donde comprobar las formas, las posiciones, las distancias, las cantidades, las calidades sensoriales y las magnitudes como el peso y el volumen.

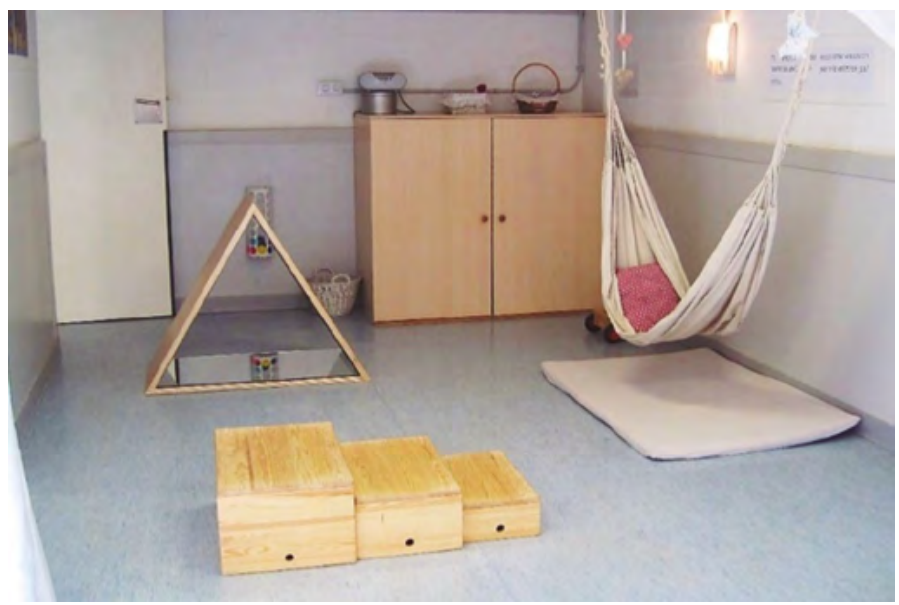

Fuente: Elaboración propia

Figura 7. Espacio de movimiento

- Los espacios de taller (Figura 8): espacios con diferentes soportes para la creación, como por ejemplo mesas, caballitos de pintura de madera o de metacrilato (para ver la translucidez de los materiales), soportes gigantes en horizontal o vertical para trazar líneas o dibujos en gran formato y en diferentes posiciones. Se incluyen también utensilios y materiales muy diversos, como pinturas de diferentes composiciones y diferentes colores para pintar con los dedos o con pincel, barro (blanco, negro y marrón), papeles de diferentes tamaños, colores y texturas, cartones, cartulinas, ceras duras, ceras blandas, tizas, rotuladores y pinceles de diferentes grosores, elementos para incrustar, moldear, recortar, sesgar, etc. En estos espacios, los niños y las niñas pueden expe- 
rimentar con el mayor número de elementos posibles, creando y formulando hipótesis e investigando sobre las diferentes posibilidades de creación, aproximándoles al despertar de los sentidos.

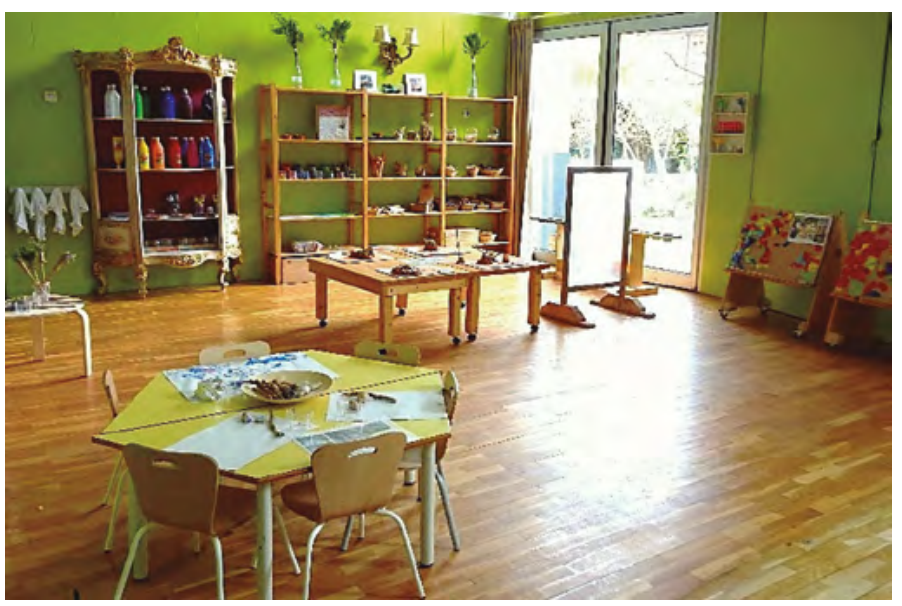

Fuente: Elaboración propia

Figura 8. Espacio de taller

- Los espacios de construcciones (Figura 9): formados por una gran cantidad de piezas de madera lisas y de formas geométricas regulares, clasificadas según un criterio específico como puede ser la forma o con alguna composición elaborada por el adulto o de un juego iniciado. Una de las características fundamentales es que, normalmente, aunque se presentan variaciones según los intereses del profesional que diseña el espacio, todas las piezas del material (menos la más pequeña) pueden componerse utilizando otras piezas más pequeñas del propio material. También existe la posibilidad de presentar piezas grandes para fomentar las construcciones colaborativas o piezas pequeñas que fomentan más el juego individual. Las posibilidades de construcción en la Escuela Infantil se inician con la formación de pilas en vertical u horizontal, la creación de puentes, los cerramientos $\mathrm{y}$, por último, las estructuras simétricas.

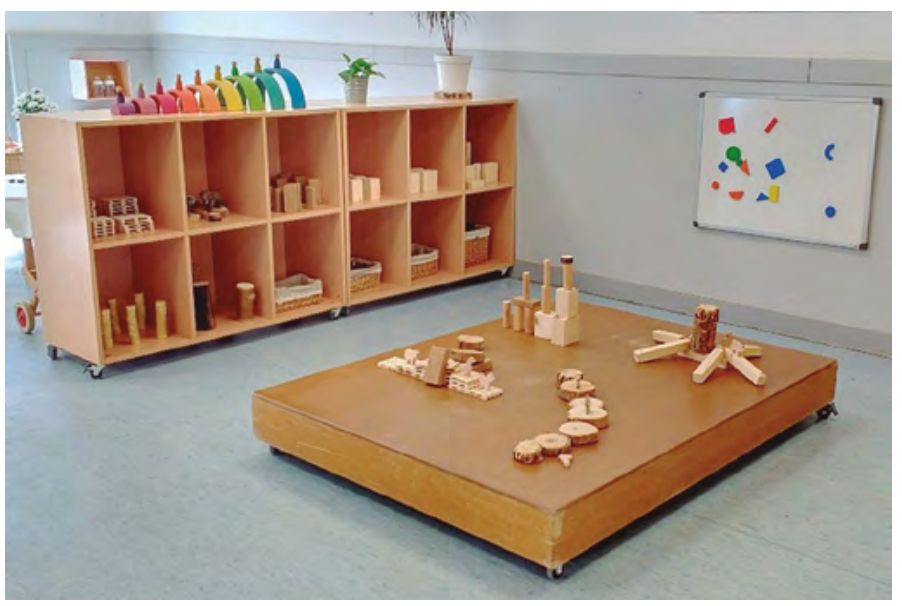

Fuente: Elaboración propia

Figura 9. Espacio de construcciones

- Los espacios con instalaciones artísticas (Figura 10): se trata de espacios que se inspiran en la corriente Land Art de arte contem- poráneo, que se basa en la creación de obras en plena naturaleza utilizando (casi siempre) los materiales que encontramos en ella (palos, piedras, etc.). En la Escuela Infantil, pueden diseñarse en el interior de las Escuelas Infantiles, pero, esta vez, en los patios y jardines exteriores.

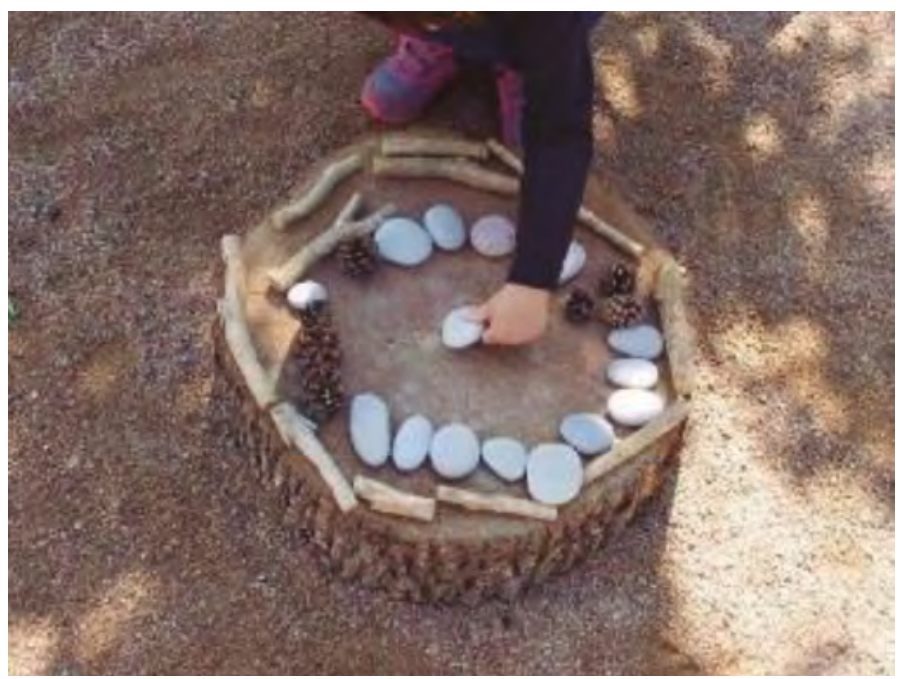

Fuente: Elaboración propia

Figura 10. Espacio de instalaciones artísticas (Land Art)

Con base en estos antecedentes, el objetivo de este estudio es analizar los conocimientos matemáticos de 28 profesionales de la Red de Escuelas Municipales de Vic para favorecer el desarrollo de los primeros conocimientos matemáticos intuitivos e informales de los niños y niñas de 0 a 3 años, a través del diseño de los espacios y materiales.

\section{Método}

Se ha diseñado un estudio cuasi-experimental (Creswell, 2009) en el que, antes y después de una actividad de formación, se han analizado los conocimientos matemáticos de 28 profesionales de la Red de Escuelas Infantiles Municipales de Vic-EBMV (Catalunya, España).

Muestra

La selección de la muestra se ha determinado por conveniencia, no aleatoria, concretamente, han participado 16 maestras, 1 maestro y 11 técnicas de Educación Infantil, de entre 23 y 60 años.

\section{Instrumentos}

Para la obtención de los datos, se ha diseñado y validado el Cuestionario "Conocimientos Didáctico-Matemáticos en la Escuela Infantil" (CDM 0-3). El tipo de cuestionario es personal, normalizado, con presencia del encuestador y semiestructurado (Anexo 1). Para determinar el nivel de conocimiento didáctico y disciplinar de los profesionales de las Escuelas Infantiles se han formulado dos tipos de preguntas: preguntas cerradas, para controlar las variables (bloque $1 \mathrm{del}$ cuestionario); y preguntas abiertas, para determinar los conocimientos didácticos y disciplinares (bloque 2 del cuestionario). Ambas han permitido obtener información de manera estructurada, minimizando el error, partiendo de un orden, con un mismo nivel de preguntas, en igualdad de condiciones y con el mismo formato (se puede consultar el cuestionario en el anexo 1). 


\section{Procedimiento}

En concreto, se han seguido las siguientes fases:

Fase 1: a través de la administración del Cuestionario CDM 0-3, se determinan los conocimientos iniciales de los profesionales de los diferentes equipos de las EBMV y se relacionan con las propuestas que ofrecían a los niños y las niñas a través de los espacios y materiales. La selección de los espacios y materiales se centra en los ambientes de juego planteados previamente acorde con las aportaciones del marco teórico: los espacios de exploración, de juego heurístico, de experimentación, de juego simbólico, de movimiento, de taller, de construcción y los espacios con instalaciones artísticas.

Fase 2: a partir de los datos, se diseña una formación para proporcionar los conocimientos necesarios para seguir avanzando en el diseño de los espacios y materiales con contenido matemático. La actividad formativa, denominada "Observación, documentación e interpretación de acciones matemáticas en la Escuela Infantil (0-3 años)", que ha ido a cargo del segundo autor del estudio, presenta los objetivos y contenidos siguientes: 1) aprender a observar, documentar e interpretar acciones matemáticas de los niños de 0 a 3 años; 2) profundizar en el significado actual de la educación matemática infantil y las principales líneas de innovación; 3) conocer las orientaciones curriculares acerca de las matemáticas de Educación Infantil y dominar los contenidos que lo integran; y 4) contemplar las nociones básicas de Didáctica de las Matemáticas en relación con la propia especialidad.

La formación comprendía 15 horas de formación, 9 presenciales y 6 no presenciales. De forma presencial, se llevaron a cabo tres sesiones de tres horas cada una: las dos primeras sesiones relacionaban la práctica educativa con la teoría propia de la disciplina. En ellas se presentaban los contenidos propios de la formación, pero a la vez, se analizaban las prácticas educativas de la Red de Escuelas Infantiles Municipales de Girona-EBMG, como ejemplo para la identificación de los contenidos del curso.

Fase 3: Una vez presentados los diferentes contenidos de formación, los profesionales han desarrollado el trabajo de manera no presencial, y su tarea ha consistido en: a) conceptualizar las acciones matemáticas presentes en las propuestas que ya se llevaban a cabo (porque muchas veces se desconocía la matemática implícita de estas propuestas que se presentaban a los niños y las niñas); y b) enriquecer estos espacios con contenido matemático y/o crear nuevos materiales y propuestas que favorecieran el desarrollo del pensamiento matemático en estas primeras edades.

En la tercera sesión de formación, eminentemente práctica, los profesionales mostraron el análisis de sus prácticas a través de presentaciones con imágenes de los diferentes espacios y materiales y, también, presentaron los cambios en los materiales y espacios, así como nuevos materiales creados.

\section{Resultados}

Se presentan los primeros resultados obtenidos a partir del Cuestionario CDM 0-3. En la Tabla 1 se presentan los datos obtenidos acerca de los Conocimientos Matemáticos Intuitivos e Informales (C-IeI), antes y después de la fase formativa respectivamente.

Tal como se observa en la Tabla 1, se producen cambios destacables en los Conocimientos Matemáticos Intuitivos e Informales (C-IeI) antes y después de la formación. En concreto, se observa que, antes de la formación, el 71,4\% de los profesionales no conocen el concepto de matemáticas informales; mientras que, después de la formación, un 89,3\% las conocen.

En la misma línea, en la Tabla 2 se presentan los primeros datos generales acerca del Conocimiento de los contenidos matemáticos (C-CM), que se ha analizado a partir de los contenidos matemáticos que consideran los profesionales en sus programaciones.

En la Tabla 2 se observa que, antes de la formación y de conocer qué son las matemáticas informales y los contenidos que las integran, en un $67,9 \%$ de las programaciones de los espacios y materiales aparecían contenidos matemáticos; mientras que, después de la formación, los contenidos matemáticos aparecen en el 100\% de las programaciones.

Además, como muestran los resultados de la Tabla 3, se evidencia también que los profesionales identifican un número mayor de contenidos matemáticos a ofrecer a los niños y a las niñas a través de las propuestas de espacios y materiales después de la formación. En este sentido, en uno de los ítems del Cuestionario CDM 0-3, los profesionales tenían que analizar los contenidos matemáticos propios que aparecían en los diferentes espacios de la Escuela Infantil descritos anteriormente. Para ello, se proporcionaron aleatoriamente tres imágenes diferentes a cada participante correspondientes a los diversos espacios de experimentación y juego (Land Art, taller, juego heurístico, movimiento, construcción, mesa de experimentación, exploración y juego simbólico).

Tabla 1. Conocimientos de los profesionales en relación con las matemáticas informales en la Escuela Infantil

\begin{tabular}{|c|c|c|c|c|}
\hline & & \multicolumn{2}{|c|}{ Después de la formación [N(\%)] } & \multirow[b]{2}{*}{ Total } \\
\hline & & Sí, conoce & No conoce & \\
\hline \multirow{2}{*}{$\begin{array}{l}\text { Antes de la formación } \\
\qquad[\mathrm{N}(\%)]\end{array}$} & Sí, conoce & $5(17,6)$ & $0(0,0)$ & $5(17,6)$ \\
\hline & No conoce & $20(71,4)$ & $3(10,7)$ & $23(82,1)$ \\
\hline & & $25(89,3)$ & $3(10,7)$ & $28(100)$ \\
\hline
\end{tabular}

Tabla 2. Contenidos matemáticos en las programaciones de los espacios y los materiales en la Escuela Infantil

\begin{tabular}{|c|c|c|c|c|}
\hline & & \multicolumn{2}{|c|}{ Después de la formación [N(\%)] } & \multirow[b]{2}{*}{ Total } \\
\hline & & Sí, presenta & No presenta & \\
\hline \multirow{2}{*}{$\begin{array}{c}\text { Antes de la formación } \\
{[\mathrm{N}(\%)]}\end{array}$} & Sí, presenta & $19(67,6)$ & $0(0,0)$ & $19(67,6)$ \\
\hline & No presenta & $9(32,1)$ & $0(0,0)$ & $9(32,1)$ \\
\hline & & $28(100)$ & $0(0,0)$ & $28(100)$ \\
\hline
\end{tabular}


Tabla 3. Media de contenidos detectados en los espacios de las Escuelas Infantiles

\begin{tabular}{|l|c|c|c|c|c|c|c|c|}
\cline { 2 - 11 } \multicolumn{1}{c|}{} & \multicolumn{9}{|c|}{ Pre } & \multicolumn{4}{c|}{ Post } \\
\cline { 2 - 11 } & $\mathrm{N}$ & Media & $\begin{array}{c}\text { Desviación } \\
\text { estándar }\end{array}$ & Mediana & $\mathrm{N}$ & Media & $\begin{array}{c}\text { Desviación } \\
\text { Estándar }\end{array}$ & Mediana \\
\hline Land art & 9 & 4,11 & 1,36 & 4 & 10 & 6,00 & 4,06 & 4,5 \\
\hline Taller & 9 & 3,78 & 1,30 & 4 & 11 & 4,09 & 2,43 & 4 \\
\hline Heuristico & 18 & 5,11 & 1,53 & 5 & 20 & 5,65 & 3,83 & 4,5 \\
\hline Movimiento & 10 & 2,00 & 1,41 & 1 & 8 & 3,13 & 1,13 & 3 \\
\hline Simbólico & 9 & 5,22 & 1,39 & 5 & 10 & 4,60 & 1,78 & 5 \\
\hline Construcción & 10 & 5,10 & 1,91 & 5 & 2 & 1,00 &, 00 & 1 \\
\hline Experimentación & 10 & 3,10 & 1,73 & 3 & 8 & 5,38 & 3,02 & 4,5 \\
\hline Exploración & 10 & 3,90 & 1,45 & 4 & 11 & 4,45 & 1,86 & 4 \\
\hline \multicolumn{1}{|c|}{ P-valor } & & & 0,000 & & & & 0,097 & 4 \\
\hline
\end{tabular}

Nota: p-valor calculado a través de Kruskal-Wallis con un nivel de confianza del 95\%

Tabla 4. Número de contenidos diseñados respecto al aprendizaje de las matemáticas en la Escuela Infantil

\begin{tabular}{|c|c|c|c|c|c|c|c|c|}
\cline { 2 - 9 } \multicolumn{1}{c|}{} & $\mathrm{N}$ & Min. & Pct 25 & Media & Mediana & Pct 75 & Máx. & $\begin{array}{c}\text { Desviación } \\
\text { estándar }\end{array}$ \\
\hline Pre & 28 & 0 & 0 & 1,3 & 1 & 2 & 4 & 1,2 \\
\hline Post & 28 & 1 & 2 & 3,5 & 3 & 5 & 8 & 1,8 \\
\hline
\end{tabular}

Nota: $\mathrm{p}$-valor $=0,000$ calculado mediante T-Student para muestras apareadas con un nivel de confianza del 95\%

Con la finalidad de no sesgar ninguna aportación, aunque la literatura muestra que el diseño de los espacios y materiales condicionan, en cierto modo, las acciones matemáticas que pueden llegar a desarrollar los niños y las niñas, se consideran todos los contenidos indistintamente y con independencia de su relevancia en todos los espacios de jugo y experimentación (álgebra temprana, números y operaciones, geometría y medida).

Tal como se muestra en la Tabla 3, la cantidad media de contenidos matemáticos aumenta en casi todos los espacios, excepto en el espacio de construcción, que podría ser debido a la escasa muestra (en el pre-test había 10 profesionales haciendo este análisis y en el post-test había 2 profesionales) y en el espacio de juego simbólico, aunque en el análisis post-test hay un profesional más, los contenidos bajan ligeramente. Sin embargo, cabe destacar que el aumento, en términos generales, se da en todos los espacios e, incluso, en el espacio de experimentación donde también baja ligeramente la muestra y el aumento continúa siendo bastante significativo.

Existen diferencias estadísticamente significativas entre los contenidos detectados por espacios antes de la formación en tanto que hay espacios donde la mediana es significativamente más alta. Concretamente, el espacio de movimiento y experimentación, antes de la formación, tienen una mediana de 1 y 3 respectivamente, mientras que los espacios de juego heurístico, construcción y simbólico, tienen una mediana de 5 .

Después de la formación, la mediana, aunque muestra una cierta tendencia a aumentar, no presenta cambios estadísticamente significativos entre los contenidos detectados en los diferentes espacios, excepto el espacio de construcciones donde baja significativamente la muestra.
En la Tabla 4 se presentan los datos obtenidos en relación con el número de contenidos matemáticos diseñados para promover el aprendizaje de las matemáticas antes y después de la formación recibida.

En términos globales, pues, existen diferencias estadísticamente significativas entre el primer y el segundo cuestionario, siendo el primero de media 1,3 respecto al segundo de 3,5.

\section{Consideraciones finales}

En este estudio se ha llevado a cabo un primer análisis de los conocimientos matemáticos del profesorado de la Escuela Infantil para favorecer el desarrollo de los primeros conocimientos matemáticos intuitivos e informales de los niños y niñas de 0 a 3 años, a través del diseño de los espacios y materiales.

Los resultados globales en relación con los contenidos diseñados en los diferentes espacios muestran cambios significativos entre antes y después de la formación. Este dato se apoya en las aportaciones de diferentes autores (Alsina, 2015; de Castro y Quiles 2014; Clements y Sarama, 2015; Geist, 2014; NCTM, 2003) que destacan la necesidad de formar a los profesionales, mostrándoles la importancia de estas primeras matemáticas.

En este sentido, los resultados muestran que los conocimientos de los profesionales acerca de las matemáticas intuitivas antes de la formación eran inferiores que después de la formación. Por ello, se considera que los conocimientos de los profesionales están estrechamente vinculados a la formación, en tanto que se ha producido un cambio significativo a partir de la actividad formativa recibida tanto en el diseño de los espacios con mayor presencia de 
contenidos matemáticos, así como en relación con los conocimientos sobre las matemáticas intuitivas correspondientes al ciclo 0-3.

Los resultados también muestran que existe una relación entre el conocimiento de los profesionales y el diseño de los espacios. Los conocimientos matemáticos de los profesionales determinan el diseño de los espacios y los materiales que ofrecen a los niños y niñas. En este sentido, los resultados presentan una diferencia significativa entre los espacios antes de la formación, donde había espacios con pocos o muy pocos contenidos matemáticos. Después de la formación, esta diferencia entre los espacios con contenido matemático tiende a desaparecer, no destacándose diferencias estadísticamente significativas entre los contenidos diseñados en los diferentes espacios. De este modo, se concluye que, si los profesionales no conocen los conocimientos matemáticos que se pueden desarrollar en la Escuela Infantil, difícilmente podrán ofrecer a los niños y a las niñas propuestas ricas y de calidad que contribuyan al desarrollo del pensamiento matemático. En este sentido, múltiples autores subrayan la necesidad de contar con profesionales formados y conscientes de la importancia de ofrecer contextos educativos de calidad que promuevan el desarrollo de acciones asociadas a las primeras matemáticas (Alsina, 2006; Alsina, 2015; Alsina y Berciano, 2018; Alsina y León, 2016; Alsina y Martínez, 2016; Björklund y Barendregt, 2016; Clements y Sarama, 2015; Edo, 2012; Varol et al., 2012).

En futuros estudios será necesario analizar de forma más minuciosa los distintos contenidos matemáticos (cualidades sensoriales, cantidades discretas, posiciones y formas y atributos mesurables) para promover el aprendizaje de las matemáticas a través de los diversos espacios y materiales, ya que en algunos estudios se ha empezado a identificar que los diversos espacios y materiales pueden potenciar distintas acciones matemáticas (Alsina y Martínez, 2016).

En síntesis, para que los profesionales puedan disponer de estos conocimientos, es imprescindible seguir investigando y que los resultados de estas investigaciones contribuyan a enriquecer la educación matemática infantil de modo que, tanto en el marco de la formación inicial como de la formación permanente, se pueda ofrecer una formación rigurosa y de calidad acerca de las matemáticas y su didáctica en la Escuela Infantil (0-3 años). Ello, además, implica avanzar necesariamente hacia una formación paritaria relativa a los dos ciclos que componen la Educación Infantil de manera que, por un lado, los estudios del Grado de Educación Infantil promuevan la competencia profesional de los futuros maestros y las futuras maestras en toda la etapa (y no sólo en el segundo ciclo) y que las Administraciones educativas se comprometan a garantizar una formación permanente de calidad a los profesionales de las Escuelas Infantiles, tal como requieren las directrices de la Unión Europea (Consejo de la Unión Europea, 2006, 2011).

\section{Agradecimientos}

Esta investigación no ha sido financiada por ninguna entidad.

\section{Contribución individual de los autores y autoras al manuscrito}

GOM ha diseñado el estudio y ha redactado el marco teórico, la metodología y los resultados. AA ha supervisado el diseño del estudio, ha redactado la introducción y las consideraciones finales y ha revisado el resto de secciones.

\section{Referencias}

Abad, J. (2006). La escuela como ámbito estético según la pedagogía reggiana. Revista Aula de Infantil, 1, 10-16.

Alsina, Á. (2006). Cómo desarrollar el pensamiento matemático de 0 a 6 años. Editorial Octaedro-Eumo.
Alsina, Á. (2012). Más allá de los contenidos, los procesos matemáticos en Educación Infantil. EDMA 0-6: Educación Matemática en la Infancia, 1(1), 1-14.

Alsina, Á. (2015). Matemáticas intuitivas e informales de 0 a 3 años: Elementos para empezar bien. Narcea Ediciones.

Alsina, Á. (2019). La educación matemática infantil en España: ¿qué falta por hacer? NÚMEROS. Revista de Didáctica de las Matemáticas, 100, 187-192.

Alsina, Á. (2020a). La Matemática y su didáctica en la formación de maestros de Educación Infantil en España: crónica de una ausencia anunciada. La Gaceta de la Real Sociedad Matemática Española, 23(2), 373-387

Alsina, Á. (2020b). Revisando la educación matemática infantil: una contribución al Libro Blanco de las Matemáticas. Edma 0-6: Educación Matemática en la Infancia, 9(2), 1-20.

Alsina, Á. y Berciano, A. (2018). Developing informal mathematics in Early Childhood Education. Early Child Development and Care, 190(13). https://doi.org/10.1080/03004430.2018.1555823

Alsina, Á. y Delgado, R. (2021). Identificando los conocimientos para enseñar matemáticas en educación infantil: un primer paso para el desarrollo profesional. Revista Sergipana de Matemática e Educação Matemática, 6(2), 1-23. https://doi. org/10.34179/revisem.v6i2.16003

Alsina, Á. y Martínez, M. (2016). La adquisición de conocimientos matemáticos intuitivos e informales en la Escuela Infantil: el papel de los materiales manipulativos. RELAdEI, Revista Latinoamericana de Educación Infantil, 5(2), 127-136.

Alsina, Á., y León, N. (2016). Acciones matemáticas de 0 a 3 años a partir de instalaciones artísticas. Educatio Siglo XXI, 34, 3362. https://doi.org/10.6018/j/263801

Azkona, J. M., y Hoyuelos, A. (2011). Reflexiones sobre la calidad en las escuelas infantiles. Tarbiya, revista de Investigación e Innovación Educativa, 42, 157-186.

Ball, D., Thames, M. H., y Phelps, G. (2008). Content knowledge for teaching: What makes it special? Journal of Teacher Education, 59(5), 389-407. https://doi.org/10.1177/0022487108324554

Baroody, A. (1987). Children's Mathematical Thinking. A developmental framework for preschool, primary, and special education teachers. Teachers College Press.

Bassedas, E., Huguet, T. y Solé, I. (2006). Aprendre i ensenyar a l'educació infantil. Graó Editorial.

Björklund, C., y Barendregt, W. (2016). Teachers' pedagogical mathematical awareness in Swedish early childhood education. Scandinavian Journal of Educational Research, 60(3), 359-377. https://doi.org/10.1080/00313831.2015.1066426

Bueno, D. (2019). Neurociencia para educadores. Ediciones Octaedro.

Carrillo, J., Climent, N., Montes, M., Contreras, L., Flores-Medrano, E., Escudero-Ávila, D., Vasco, D., Rojas, N., Flores, P., Aguilar-González, Á., Ribeiro, M., y Muñoz-Catalán, Mª . C. (2018). The mathematics teacher's specialised knowledge (MTSK) model. Research in Mathematics Education, 20, 236-253. https://doi.org/10.1080/14794802.2018.1479981

Castro, E. y Castro, E. (Eds.) (2016). Enseñanza y aprendizaje de las matemáticas en Educación Infantil. Pirámide.

Charalambous, C., y Pitta-Pantazi, D. (2016). Perspectives on priority mathematics education: Unpacking and understanding a complex relationship linking teacher knowledge, teaching, and learning. En L. English y D. Kirshner (Eds.), Handbook of International Research in Mathematics Education (pp. 19-59). Routledge.

Clements, H.D., y Sarama J. (2015). El aprendizaje y la enseñanza de las matemáticas. El enfoque de las Trayectorias de Aprendizaje. Learning Tools LLC.

Conclusiones del Consejo y de los Representantes de los Gobiernos de los Estados miembros, reunidos en el seno del Consejo, sobre eficiencia y equidad en educación y formación (2006). Diario Oficial de la Unión Europea, C 298/3, 8.12.2006, 3-6. ht- 
tps://eur-lex.europa.eu/legal-content/ES/TXT/PDF/?uri=CELEX:42006X1208(01)\&from=ES

Conclusiones del Consejo sobre educación infantil y atención a la infancia: ofrecer a todos los niños la mejor preparación para el mundo de mañana (2011). Diario Oficial de la Unión Europea, C 175/03, 15.06.2011, 8-10. http://waece.org/xxxxx.pdf

Creswell, J. W. (2009). Research design: Qualitative, quantitative, and mixed methods approaches. SAGE Publications, Inc.

de Castro, C. (2011). Buscando el origen de la actividad matemática: Estudio exploratorio sobre el juego de construcción infantil. Escuela Abierta, 14, 47-65.

de Castro, C., Flecha, G., y Ramírez, M. (2015). Matemáticas con dos años: buscando teorías para interpretar la actividad infantil y las prácticas docentes. Tendencias Pedagógicas, 26, 89-108.

de Castro, C., González, A., y Escorial, B. (2009). El aprendizaje de las matemáticas a los tres años: Narración reflexiva sobre la construcción de un mercado medieval. Números: Revista de Didáctica de las Matemáticas, 70, 53-65.

de Castro, C., y Quiles, Ó. (2014). Construcciones simétricas con 2 y 3 años: La actividad matemática emergente del juego infantil. Aula de Infantil, 77, 32-36.

Edo, M. (2012). Ahí empieza todo. Las matemáticas de cero a tres años. Números: Revista de Didáctica de las Matemáticas, 80, 71-84.

Galardini, A. L. (2010). Entrevista a Anna Lia Galardini. Cuadernos de Pedagogía, 397, 61-64.

Geist, E. (2014). Children are born mathematicians: supporting mathematical development, birth to age 8. Pearson.
Godino, J. D., Giacomone, B., Batanero, C., y Font, V. (2017). Enfoque ontosemiótico de los conocimientos y competencias del profesor de matemáticas. Bolema: Boletim de Educação Matemática, 31(57), 90-113. https://doi.org/10.1590/1980-4415v31n57a05

Goldschmied, E., y Jackson, S. (2007). La educación infantil de 0 a 3 años. Morata.

Hoyuelos, A. (2010). La identidad de la educación infantil. Educação, 35(1), 15-23.

Jubete, M. (Ed.). (2004). Espais i temps per al joc. Rosa Sensat.

Malaguzzi, L. (2001). La educación infantil en Reggio Emilia. Octaedro.

National Council of Teachers of Mathematics [NCTM]. (2003). Principios y Estándares para la Educación Matemática. National Council of Teachers of Mathematics (traducción de la Sociedad Andaluza de Educación Matemática THALES).

Rinaldi, C. (2009). In dialogo con Reggio Emilia: ascoltare, ricercare e apprendere: discorsi e interventi 1984-2007. Reggio Children.

UNICEF (2017). La primera infancia importa para cada niño. Recuperado de https://www.unicef.org/peru/sites/unicef.org.peru/ files/2019-01/La_primera_infancia_importa_para_cada_nino_ UNICEF.pdf

Varol, F., Farran, D. C., Bilbrey, C., Vorhaus, E. A., y Hofer, K. G. (2012). Improving mathematics instruction for early childhood teachers: Professional development components that work. NHSA Dialog, 15(1), 24-40. https://doi.org/10.1080/152 40754.2011.636488

Vila, B. (2018). Amb els infants i per als infants. Infància: educar de 0 a 6 anys, 223, 4-8. 
Anexo 1: Cuestionario "CDM-MAT0-3"

\section{Cuestionario “CDM-MAT0-3"}

Instrumento de recogida de información sobre los conocimientos didáctico-disciplinares, los comportamientos y la valoración de los profesionales de la Escuela Infantil en relación a las matemáticas informales para el diseño de los espacios y materiales en la Escuela Infantil para la Tesis Doctoral" Espacios, materiales y desarrollo de las matemáticas informales en la Escuela Infantil"

Por favor, contesta las preguntas que se detallan a continuación en silencio y si tiene alguna duda diríjase al encuestador.

\section{Bloque 1. Datos generales}

1. Identifica la fecha y la hora:

2. Identifica el tipo de contratación laboral
a. Personal en plantilla
b. Personal en substitución

3. Marca el aula de referencia del curso 2016-17:
Lactantes $\square$
$1-2$ 口
2-3ㅁ
Heterogéneo $\square$

4. Sexo (marca con una X): Femenino () Masculino ()

5. ¿Cuál es tu título profesional?

6. ¿Cuán preparado te sientes para diseñar los espacios y materiales bajo criterios matemáticos? (marque con una X)

Muy preparado

Medianamente preparado

Poco preparado

No me siento preparado

7. ¿En el diseño de los espacios y materiales que planificas presentas de manera consciente contenidos matemáticos con el objetivo de acercar estos contenidos a los alumnos? (marque con una $\mathrm{X}$ )

Si

( )

No 
8. ¿En tu formación universitaria como maestra o de instituto como educadora tuviste cursos de didáctica de las matemáticas para el ciclo educativo 0-3? (marque con una X) $\mathrm{Si}$ ( ) No( )

9. ¿En tu formación como maestra o de instituto como educadora tuviste cursos de diseño de espacios y materiales para el ciclo educativo 0-3? (marque con una $\mathrm{X}$ )

$\begin{array}{ll}\text { Si } & \text { ( ) } \\ \text { No ( ) }\end{array}$

10. ¿Durante los años que llevas trabajando como maestra o educadora del ciclo educativo 0-3 has realizado algún curso de formación continua en didáctica de las matemáticas?
$\mathrm{Si} \quad($ )
No ()

Bloque 2.

1. ¿Has tenido la oportunidad en alguna ocasión de leer/escuchar información acerca del término "matemáticas informales"?

$\begin{array}{ll}\text { Si () } & \\ \text { No () }\end{array}$

2. En caso afirmativo, ¿podrías explicar brevemente a qué se refiere?

3. Observa los diferentes espacios y materiales que se muestran e indica los contenidos y las capacidades matemáticas que trabaja en ellos.

Identifique la selección de imágenes que se le ha asignado
a. $\mathrm{CDM}-03-\mathrm{F} 1$
( )
b. CDM-03-F2 ()
c. $\mathrm{CDM}-03-\mathrm{F3}$ () 
4. ¿En las programaciones donde diseña los espacios y materiales de tu aula se reflejan objetivos entorno al aprendizaje de las matemáticas? (marque con una X)

$\begin{array}{ll}\text { Si } & \text { ( ) } \\ \text { No ( ) }\end{array}$

5. En caso afirmativo, enumera 2 objetivos y describe 2 de las acciones matemáticas que contemplan. En caso negativo, explica los motivos 


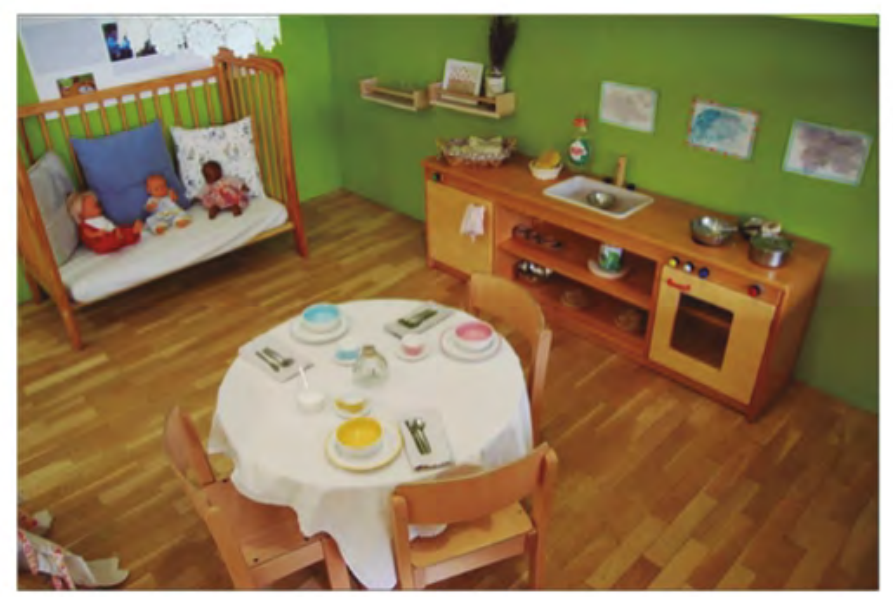

Espacio de Land Art

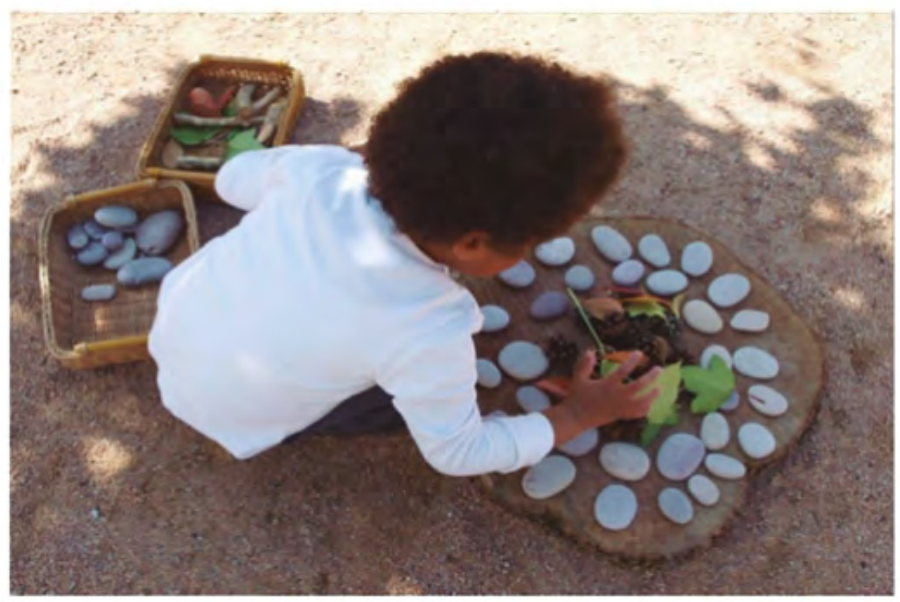

Espacio de

Juego heuristico

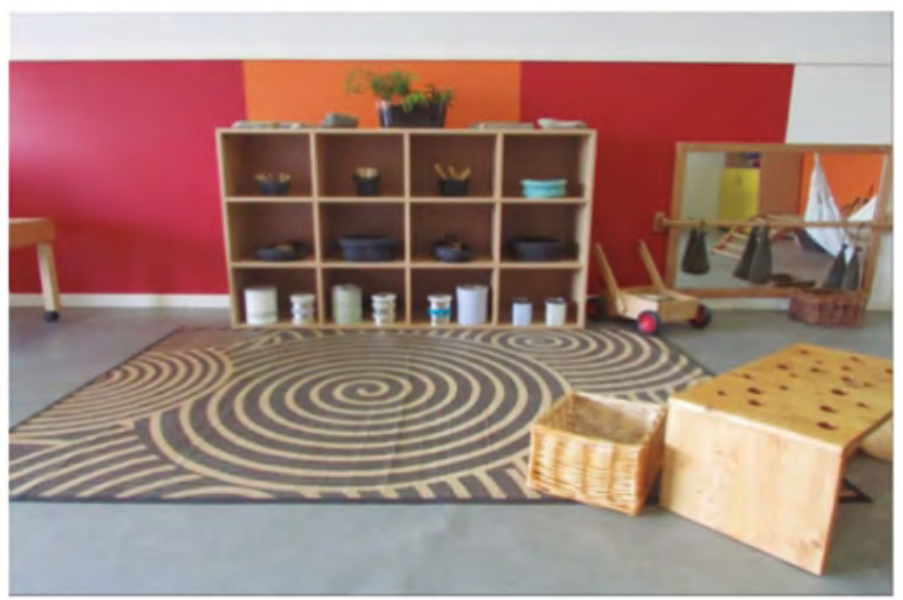

Espacio de juego simbólico 


\section{CDM-03-F2}

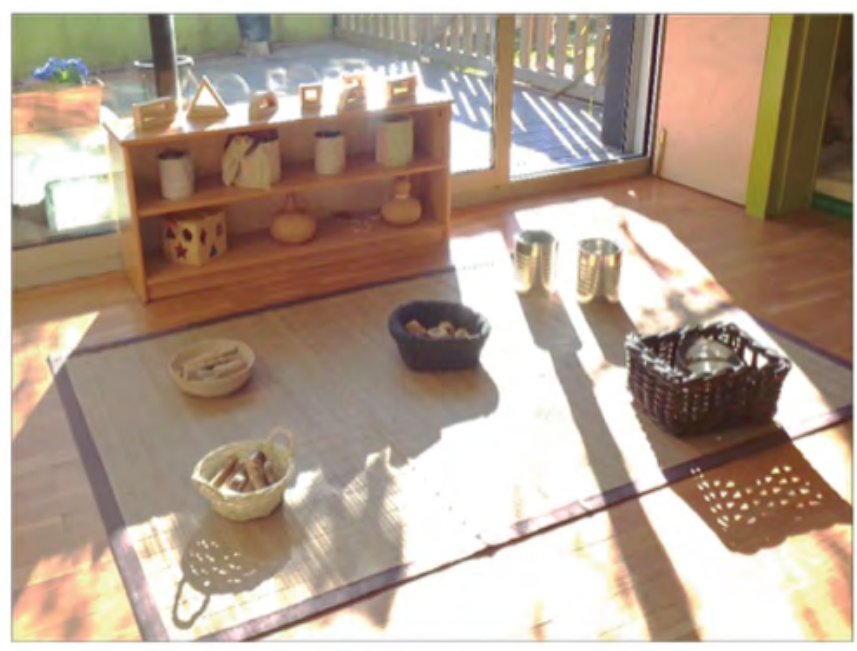

Espacio de juego heurístico

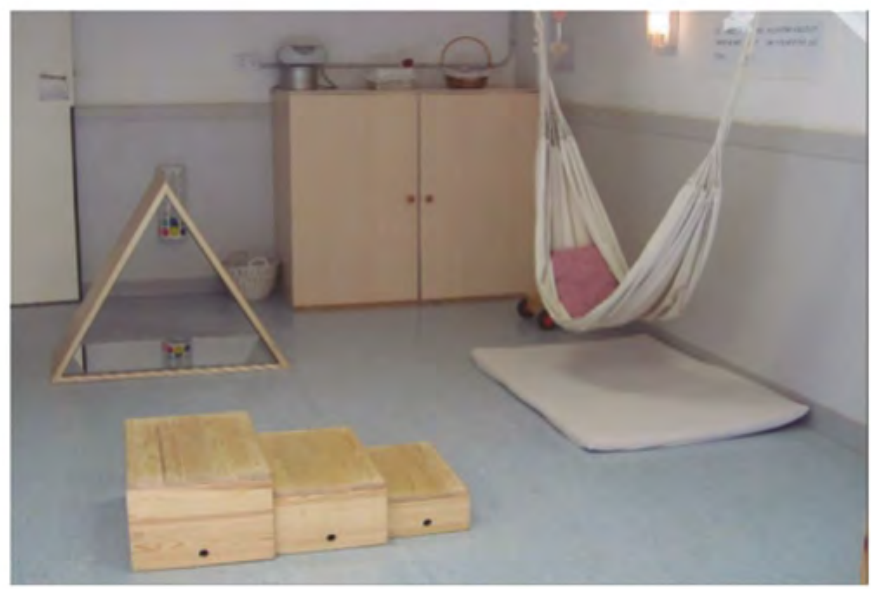

Espacio de movimiento

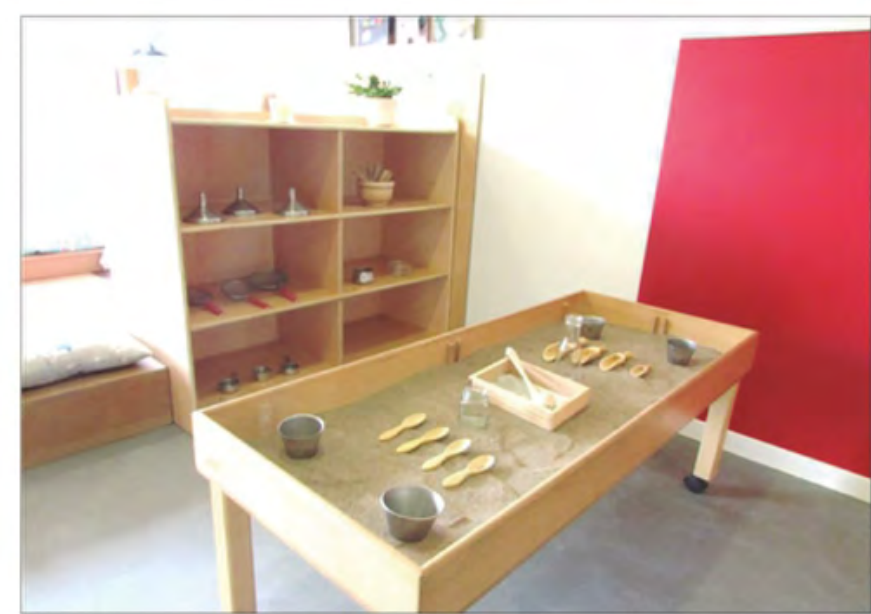

experimentación 
CDM-03-F3

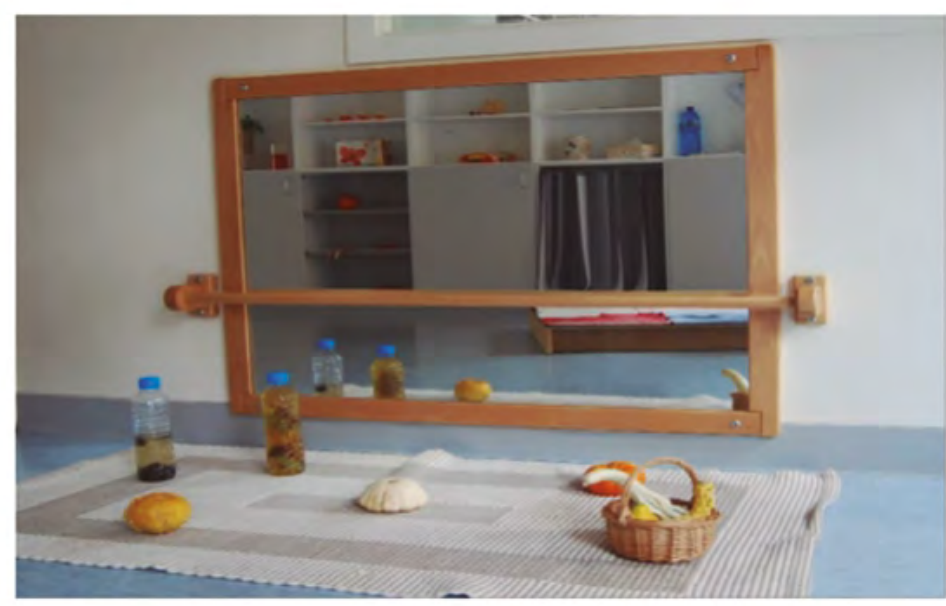

Espacio de

exploración

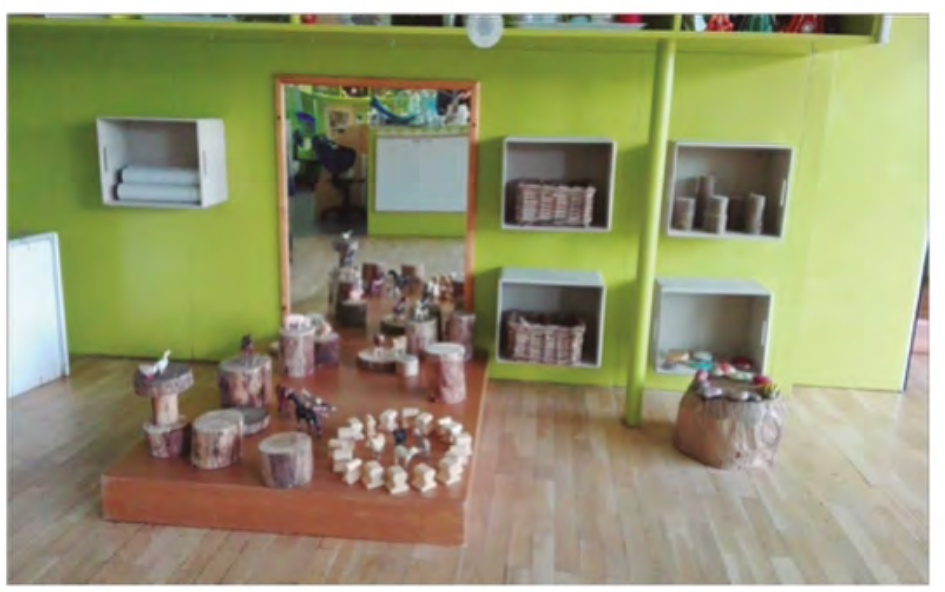

Espacio de

construcción

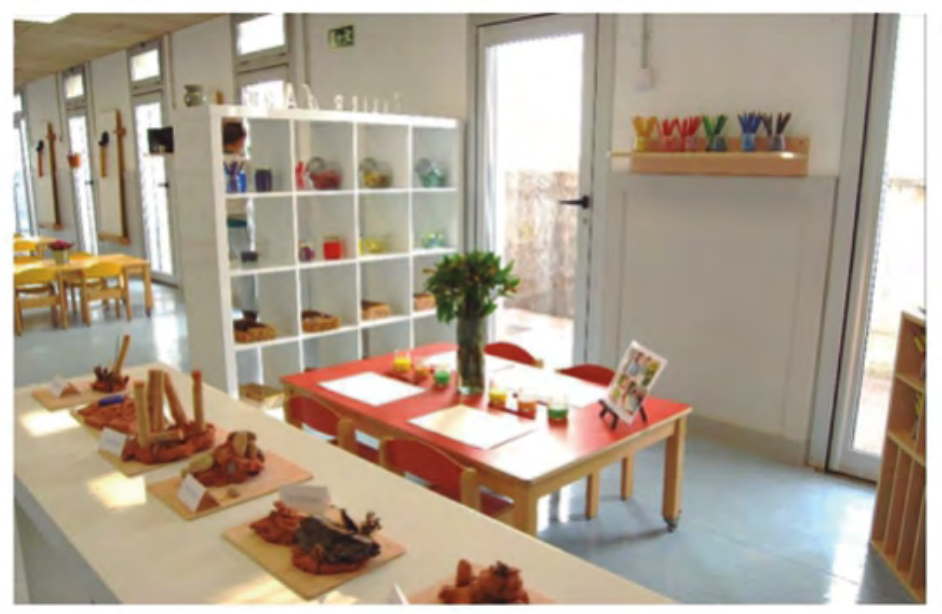

Taller 